\title{
Chiral Discrimination through Bielliptical High-Harmonic Spectroscopy
}

\author{
Denitsa Baykusheva and Hans Jakob Wörner* \\ ETH Zürich, Laboratory of Physical Chemistry, 8039 Zurich, Switzerland
}

\begin{abstract}
(Received 10 April 2018; revised manuscript received 26 July 2018; published 10 September 2018)
\end{abstract}
\begin{abstract}
Molecular chirality plays an essential role in most biochemical processes. The observation and quantification of chirality-sensitive signals, however, remains extremely challenging, especially on ultrafast timescales and in dilute media. Here, we describe the experimental realization of an all-optical and ultrafast scheme for detecting chiral dynamics in molecules. This technique is based on high-harmonic generation by a combination of two-color counterrotating femtosecond laser pulses with polarization states tunable from linear to circular. We demonstrate two different implementations of chiral-sensitive high-harmonic spectroscopy on an ensemble of randomly oriented methyloxirane molecules in the gas phase. Using two elliptically polarized fields, we observe that the ellipticities maximizing the harmonic signal reach up to $4.4 \pm 0.2 \%$ (at $17.6 \mathrm{eV}$ ). Using two circularly polarized fields, we observe circular dichroisms ranging up to $13 \pm 6 \%(28.3-33.1 \mathrm{eV})$. Our theoretical analysis confirms that the observed chiral response originates from subfemtosecond electron dynamics driven by the magnetic component of the driving laser field. This assignment is supported by the experimental observation of a strong intensity dependence of the chiral effects and its agreement with theory. We moreover report and explain a pronounced variation of the signal strength and dichroism with the driving-field ellipticities and harmonic orders. Finally, we demonstrate the sensitivity of the experimental observables to the shape of the electron hole. This technique for chiral discrimination will yield femtosecond temporal resolution when integrated in a pump-probe scheme and subfemtosecond resolution on chiral charge migration in a self-probing scheme.
\end{abstract}

DOI: 10.1103/PhysRevX.8.031060

Subject Areas: Optics

\section{INTRODUCTION}

The detection and characterization of chirality plays a central role in a broad range of sciences. Therefore, the development of ever more sensitive chiral spectroscopies has received considerable attention. Their basic principle relies on the interaction with light, which is itself chiral [e.g., circularly polarized light (CPL)] [1], as in optical rotatory dispersion [2], circular dichroism (CD), or Raman optical activity [3-6]. The asymmetry in the absorption of right versus left CPL is typically $<10^{-3}$ (see Ref. [7]) due to the small size of most chiral molecules relative to the helical pitch of the CPL [8], which has limited the applicability of these methods to the condensed phase.

Recently, several chiral-sensitive techniques have been developed for the gas phase. The sensitivity of these methods, such as three-wave-mixing microwave spectroscopy [9], Coulomb-explosion imaging [10-13], and laser-induced mass spectrometry $[14,15]$, resides in the electric-dipole

\footnotetext{
*hwoerner@ethz.ch
}

Published by the American Physical Society under the terms of the Creative Commons Attribution 4.0 International license. Further distribution of this work must maintain attribution to the author(s) and the published article's title, journal citation, and DOI. nature of the relevant interactions. Photoelectron circular dichroism (PECD) [16] encodes the chiral response into the asymmetry of the photoelectron angular distribution with respect to the field propagation direction $[17,18]$. PECD measurements have been realized both in the single-photon $[16,19]$ as well as in the multiphoton regimes [20-23] and have recently been extended to study molecular relaxation dynamics [24] and time delays in multiphoton ionization [25].

High-harmonic generation (HHG) has recently been shown to offer an attractive alternative approach [26,27]. HHG can be rationalized on a simplistic level as a sequence of three steps: strong-field ionization, propagation of the liberated electron in the continuum, and recombination with the parent ion. As each step encodes structure-specific information into the resulting spectrum, a temporal resolution of a fraction of an optical cycle $(\approx 0.1 \mathrm{fs})$ can be achieved [28-30]. Unfortunately, CD studies using HHG have remained extremely challenging due to the drastic reduction of the HHG yield associated with the increase of the driving-field ellipticity. The only published experimental investigation of chiral media using HHG [27] revealed a chiral response defined in terms of the ellipticity maximizing the HHG yield on the order of $1 \%$ for methyloxirane and $2 \%$ for fenchone.

In this article, we describe the realization of an experimental scheme for studying chiral ultrafast phenomena in 
isolated molecules. It relies on bicircular high-harmonic generation (BHHG) [31-33] driven by laser fields of tunable ellipticities. The application of BHHG to study static and time-dependent symmetries of atoms and molecules has been termed bicircular high-harmonic spectroscopy (BHHS) [34-36]. Its potential for the study of chiral molecules has ignited some theoretical interest $[26,37,38]$, whereby inconsistent predictions regarding the expected degree of circular dichroism have been made ( $3 \%$ in Ref. [37] versus up to $80 \%$ in the previous study [26]). On the experimental side, chirality-sensitive BHHS is still in development, and promising experimental results have been reported from several groups. In Ref. [39], Fleischer et al. reported on the observation of a 30\%-chiral effect. Using an experimental scheme based on the detection of photoelectrons generated in a krypton gas target employing BHHG radiation, Harada et al. [40] found a pronounced difference in the responses of the $(R)$ and the $(S)$ enantiomers of gas-phase limonene.

Our experimental results obtained with methyl oxirane $\left(\mathrm{MOX}, \mathrm{C}_{3} \mathrm{H}_{6} \mathrm{O}\right.$ ) show $\mathrm{CD}$ effects of $3 \%-8 \%$ over wide parameter ranges, reaching up to $13 \%$ in specific cases. This level of chiral discrimination is similar to that achieved in PECD, but is simpler to realize because it is detected alloptically and does not require the angle-resolved detection of electrons. Moreover, the CD observed in our work extends over a broad range of photon energies $(\sim 10-30 \mathrm{eV})$, in contrast to PECD, which is large only in the close vicinity of the ionization threshold (see, e.g., Ref. [19]). Our theoretical analysis shows that the measured $\mathrm{CD}$ is entirely dominated by laser-field-induced magnetic-dipole transitions in the molecular cation between ionization and recombination. In this way, our findings lend support to the predictions and conclusions of previous theoretical work $[26,37,38]$. The observed chiral discrimination thus relies on subfemtosecond electron dynamics driven by the magnetic-field component of the laser field, offering the opportunity to study a new class of laser-controlled charge migration [30].

\section{RESULTS}

Our experiments consist in measuring the high-harmonic spectra emitted from enantiopure samples of chiral molecules (methyl oxirane) in a seeded supersonic molecular beam. High-harmonic generation is driven by a laser field synthesized from elliptically polarized laser pulses centered at 1800 or $1900 \mathrm{~nm}$ ( 40 fs pulse duration) and their second harmonic with opposite ellipticity. The emitted highharmonic spectra, which typically cover photon energies of up to $35 \mathrm{eV}$, are detected with a flat-field spectrometer. Further experimental details are given in the Appendix.

We discuss two complementary approaches for detecting $\mathrm{CD}$ using BHHS. In both cases, the HHG yields of the two enantiomers are recorded as a function of the field ellipticity $\left(\epsilon_{\omega}=-\epsilon_{2 \omega}\right)$ as the polarizations of the fundamental $(\omega)$ and the second-harmonic $(2 \omega)$ components are simultaneously varied from linear $\left(\epsilon_{\omega}=-\epsilon_{2 \omega}=0\right)$ via elliptical to circular $\left(\epsilon_{\omega}=-\epsilon_{2 \omega}=1\right)$. The two schemes differ in the range of ellipticities at which the CD effect is studied and will be referred to as "bicircular" and "bielliptical." Details on the optical implementation of these two schemes are given in the Appendix.

Figure 1 illustrates the results of the bicircular measurements. The top panel of Fig. 1(c) displays the highharmonic spectrum emitted from $R$-MOX when using circularly polarized driving fields. This spectrum displays the expected emission of pairs of neighboring frequencies, i.e., $(3 q+1) \omega$ and $(3 q+2) \omega$, whereas the frequencies $3 q \omega$ are strongly suppressed ( $q$ being an integer). This is a consequence of the conservation of angular momentum, spin symmetry, and parity in BHHG, which has been discussed previously $[31,32,34]$.

Our work focuses on identifying the signatures of molecular chirality in these spectra. For this purpose, we show in Fig. 1(a) the intensity of a selected harmonic order (H20) emitted from $R$ - or $S$-MOX as a function of the ellipticity $\epsilon_{\omega}=-\epsilon_{2 \omega}=|\epsilon|$ of the two field components, which is varied by rotating an achromatic broadband zeroorder quarter-wave plate (QWP) in the common beam path. The relation between the ellipticity and the QWP angle $\alpha$ is given by $\epsilon=\tan (\alpha)$. The comparison of the two traces immediately reveals that the intensity of the $R$ enantiomer dominates for $\alpha=45^{\circ}$, whereas that of the $S$ enantiomer dominates for the opposite setting. Prior to comparison, the traces corresponding to the two enantiomers have been normalized with respect to the signal integrated over the entire scanned range as a function of the QWP angle $\alpha$, i.e.,

$$
I_{R / S}(\alpha, n \omega)=\frac{\tilde{I}_{R / S}(\alpha, n \omega)}{\int_{\alpha} \tilde{I}_{R / S}(\alpha, n \omega) d \alpha},
$$

with $\tilde{I}_{R / S}(\alpha, n \omega)$ being the signal prior to normalization. We note that the value of the denominator in Eq. (1) is independent of the system's chirality. We first discuss the $\mathrm{CD}$ at a given photon energy $n \omega$ as a function of $\alpha$, defined as

$$
\mathrm{CD}(n \omega, \alpha)=\frac{2\left[I_{R}(n \omega, \alpha)-I_{S}(n \omega, \alpha)\right]}{\max \left[I_{R}(n \omega, \alpha)+I_{S}(n \omega, \alpha)\right]},
$$

where max stands for the maximum with respect to $\alpha$. $\mathrm{CD}(n \omega, \alpha)$ is plotted as a green curve in Fig. 1(b). The chiral response maximizes for ellipticity values close to perfectly circular polarization. It is opposite for the two rotation directions of the light polarization, as required by symmetry. Our definition of CD differs from the traditional one, where the denominator is merely given by the sum of the signals of the two enantiomers, because the latter yields artificially high values of the $C D$ in the ellipticity regions associated with low intensities and is 

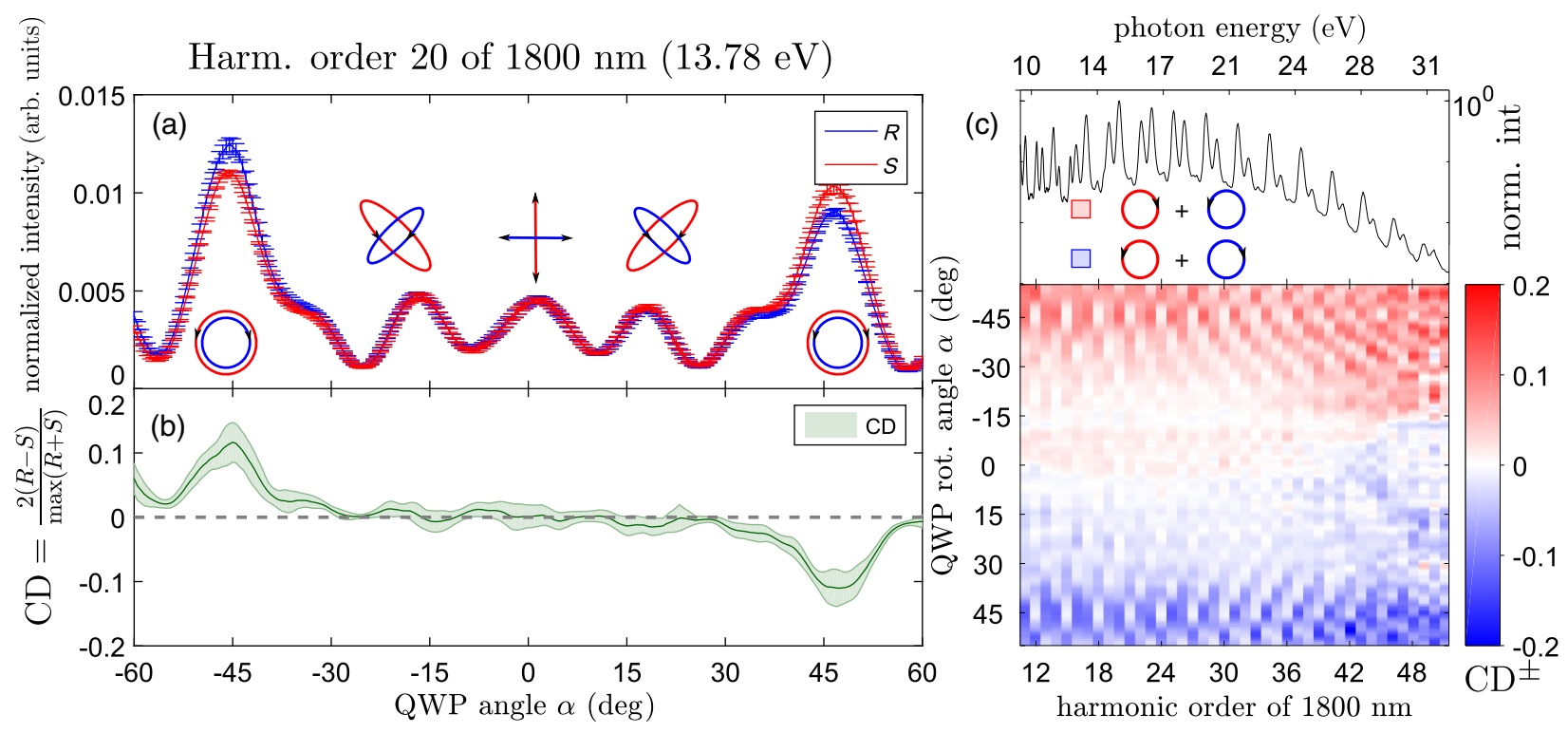

FIG. 1. Circular dichroism measurement using the bicircular scheme. (a) HHG yields for harmonic 20 of $R$ - and $S$-MOX as a function of the QWP rotation angle. A moving average with a span of 5 data points was applied on the measured signal. The insets show Lissajous representations of the electric component of the driving laser fields. (b) Ellipticity-dependent differential circular dichroism signal $\mathrm{CD}(20 \omega, \alpha)$. (c) Top: Spectrum of $R$-MOX recorded for the setting $\alpha=-45^{\circ}$; bottom: spectrogram showing the CD as a function of the QWP angle and the photon energy. The colors encode the sign of the CD. The intensities are $I_{\omega} \sim 3 \times 10^{13} \mathrm{~W} / \mathrm{cm}^{2}$ and $I_{2 \omega} \sim 1.1 \times 10^{13} \mathrm{~W} / \mathrm{cm}^{2}$. The error bars in (a) represent 2 standard deviations of the measured signal.

even ill defined in the limit of pure bicircular polarization since $I_{R / S}\left(3 q \omega, \pm 45^{\circ}\right)=0$.

The ellipticity-resolved CD signal shown in the lower panel of Fig. 1(c) exhibits a pronounced oscillatory pattern, which is mainly localized in the high-ellipticity region $\alpha \in\left[ \pm 35^{\circ}- \pm 55^{\circ}\right]$ and progressively decreases as the ellipticity is diminished. Each class of harmonic orders $[n=\{3 q,(3 q+1),(3 q+2)\}]$ possesses a distinct modulation pattern; most notably, the maxima of $\mathrm{CD}(3 q, \omega)$ are shifted from the positions of maximum field ellipticity by $\Delta \alpha \approx \pm 10^{\circ}$, whereas the remaining groups peak around $\alpha \approx \pm 45^{\circ}$. A comparison of the CD oscillation pattern with the ellipticity variation of the HHG yield is presented in Fig. 2 for three selected harmonic orders [and over the complete range of photon energies in Figs. S1(a) and S2(a) of Supplemental Material (SM) [41]]. These results show that the maxima of $|\mathrm{CD}(n \omega, \alpha)|$ closely follow the locations of maximal spectral intensity.

In the following, we use the trajectory formalism arising from the saddle-point treatment of the BHHG process [42] in order to identify the physical origin of this behavior. Restricting the analysis to one cycle of the fundamental, the suppression of the $3 q$ orders at $\alpha= \pm 45^{\circ}$ results from the destructive interference of the emission from three identical electron trajectories separated by a phase shift of $(2 \pi) /(3 \omega)$. Multiple representative "branches" of such solutions for ionization events constrained to $t_{i} \in$ $\{-[(2 \pi) /(3 \omega)], 0\}$ are given in Fig. S3 of the SM [41] as a function of the (real part of the) recombination time.
Detuning the ellipticity of the drivers from circularity perturbs the location of the saddle points and lifts the degeneracy between the three replicas of each branch [cf. Figs. S4(a) and S4(b) of SM [41]], thus removing the destructive interference in the $3 q$ harmonics. As a result, the latter start to gain intensity as the polarization of the driving fields is tuned from circular to elliptical.

We restrict our analysis to the set of shortest electron trajectories (saddle-point solutions; see red line in Fig. S3 of SM [41]). This contribution is by far the dominant one because the solutions associated with longer travel times are exponentially suppressed by wave-packet dispersion. This solution is, moreover, strongly favored by phasematching considerations. Simultaneously, we consider only small deviations from the (bi)circular case, i.e., $\Delta \alpha \approx \pm 15^{\circ}$, as in this region the spectrum can still be assumed to be dominated by this single-branch contribution. We calculate the perturbed saddle points within one cycle of the fundamental and coherently sum their contributions using a simplified approach based on hydrogenlike ionization or recombination matrix elements. Interestingly, this approximate treatment is sufficient to capture the main features of the oscillatory structure of the spectrum (cf. Fig. 2 herein and Fig. S5 of SM [41]). The experimental variations of the intensities of three selected harmonic orders as a function of the QWP angle are compared to the theoretical predictions in Figs. 2(a)-2(c). As observed in the experiment, the modifications in the relative phases between the emissions from the three subcycle replicas of 

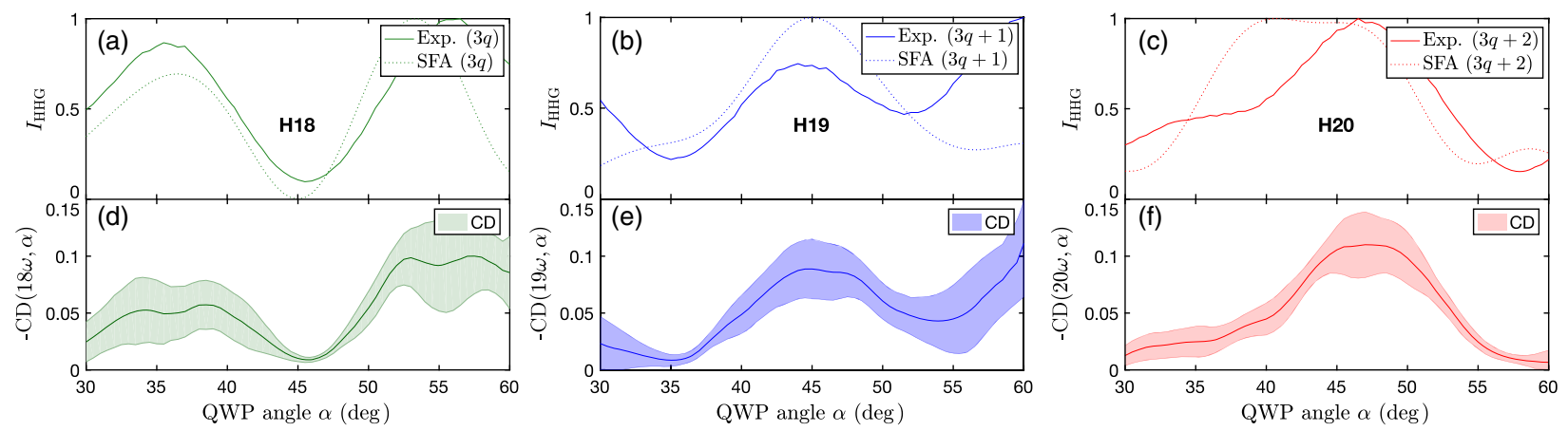

FIG. 2. Ellipticity dependence of the spectral intensity and the CD. (a)-(c) Comparison of the HHG intensity modulations for harmonic orders 18, 19, and 20 of $1800 \mathrm{~nm}$ (full lines) with the calculation results (dashed lines) obtained with the theoretical model discussed in the text. The data sets shown correspond to the measurement presented in Fig. 1 for $R$-MOX. Panels (d)-(f) show the corresponding ellipticity-dependent chiral response $\operatorname{CD}(n \omega, \alpha)$. The strong-field (SFA) calculations have been performed assuming a single-cycle continuous-wave bicircular pulse centered at $1800+900 \mathrm{~nm}$, with intensity of the fundamental driving field set at $I_{\omega}=5 \times 10^{13} \mathrm{~W} / \mathrm{cm}^{2}$ and a field strength ratio of $\eta=F_{2 \omega} / F_{\omega}=0.75$.

one trajectory branch give rise to maxima in the $3 q$ harmonic intensities appearing at $\alpha=35^{\circ}$ and $52^{\circ}$, respectively. These are the same positions at which the associated $\mathrm{CD}$ signal $(|\mathrm{CD}(3 q \omega, \alpha)|)$ maximizes, as evident from the data displayed in Figs. 2(d)-2(f). At perfect ellipticity, the destructive-interference condition brings the $\mathrm{CD}$ below the detection threshold of the experiment. Simultaneously, the introduced relative phase shift between the three emissions from the trajectories can result in destructive interferences in the remaining two groups of harmonics, as seen from the spectral signal for $n=3 q \pm 1$ at $\alpha \approx 35^{\circ}$. Importantly, the magnitude of the $\mathrm{CD}$ again closely follows the magnitude of the total signal, just as in the case of the $3 q$ harmonics. The fact that the forbidden harmonics maximize at positions different from $\alpha=45^{\circ}$ has consequences for the magnitude of the chiral response at these energies, as we elaborate below when discussing the ellipticity-averaged CD.

Our analysis thus explains the observed modulations of the high-harmonic intensities as a function of the ellipticity (or QWP angle) in terms of constructive and destructive interferences between the three dominant trajectories launched during one cycle of the fundamental field. We further observe that the magnitude of the $\mathrm{CD}$ follows the high-harmonic intensity, suggesting that the CD itself is controlled by the same interference effect that modulates the high-harmonic intensity, for both the "allowed" harmonics $(3 q \pm 1)$ and the forbidden harmonics $(3 q)$.

We now investigate the effect of the laser parameters on the observed spectra. This is an important aspect in all studies of high-harmonic spectroscopy, since it enables a distinction of effects originating from the static (electronic and geometric) structure of molecules from effects induced by dynamics taking place between ionization and recombination [43-47]. The importance of such dynamics in the chiral response has indeed been inferred from theoretical work in Ref. [27], but an experimental confirmation in terms of an intensity dependence of the observed spectra has not been reported.

Figure 3 shows two complete sets of data recorded at different intensities, i.e., an unchanged fundamental intensity $\left(I_{\omega}=3 \times 10^{13} \mathrm{~W} / \mathrm{cm}^{2}\right)$ and a second-harmonic intensity of $I_{2 \omega}=1.1 \times 10^{13} \mathrm{~W} / \mathrm{cm}^{2}$ (top row of Fig. 3, same as in Fig. 1) or $I_{2 \omega}=7.5 \times 10^{12} \mathrm{~W} / \mathrm{cm}^{2}$ (bottom row). This minor change of the intensity ratio between the two fields has a major impact on the observed signals.

First, we note that neither the cutoff nor the intensity envelope of the HHG spectra [Figs. 3(a) and 3(b)] is significantly changed. In contrast to this, the intensity ratio of neighboring harmonics $[I(3 q+2) / I(3 q+1)$, shown in Figs. 3(c) and 3(d)] changes substantially; e.g., the photon energy at which the ratio drops below 1 shifts from $\sim 16$ to $\sim 13 \mathrm{eV}[35,48]$. Second, we study the CD as a function of energy by integrating over ellipticity ranges of large CD:

$$
\overline{\mathrm{CD}}^{ \pm}(n \omega)=\int_{ \pm 20^{\circ}}^{ \pm 55^{\circ}} \mathrm{CD}(n \omega, \alpha) d \alpha
$$

The superscript \pm specifies the helicities of the two field components; i.e., "+" denotes the configuration where the fundamental beam is (close-to-)right circularly polarized $\left(\epsilon_{\omega} \approx 1\right)$ and the second harmonic is (close-to-)left circularly polarized $\left(\epsilon_{2 \omega} \approx-1\right)$ and vice versa.

The quantity $\overline{\mathrm{CD}}^{ \pm}(n \omega)$ [Figs. 3(e) and 3(f)] shows an even higher sensitivity to the intensity compared to the previously discussed observables. The reduction of $I_{2 \omega}$ by one-third reduces the $\overline{\mathrm{CD}}^{ \pm}(n \omega)$ by a factor of $\sim 2-5$, depending on the photon energy. In the SM (Sec. 2.2) [41], we present additional measurements recorded at a different fundamental wavelength $(1420 \mathrm{~nm}$, cf. Fig. S7 [41]) and two different intensities. The strong dependence of the measured $\mathrm{CD}$ on the wavelengths and intensities of the driving lasers suggests that the $\mathrm{CD}$ is dominated by 

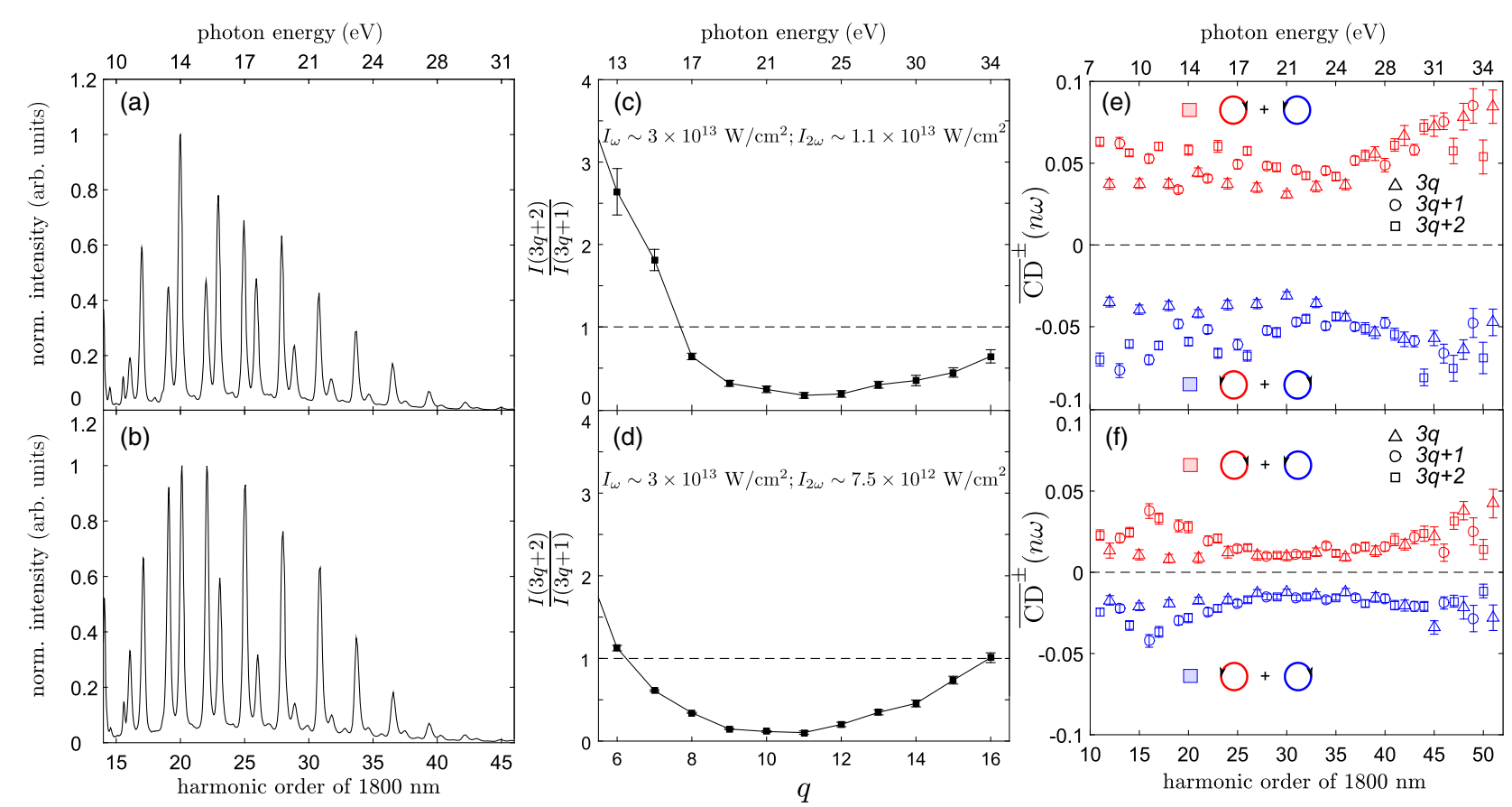

FIG. 3. Intensity dependence of CD. (a),(b) Angle-integrated $\left[\alpha \in \pm\left(20^{\circ}-55^{\circ}\right)\right] \overline{\mathrm{CD}}^{ \pm}(n \omega)$ for bielliptical fields of opposite handedness ( + or - , encoded as blue or red) as a function of the photon energy for two different intensities [cf. text insets in (c) and (d)]. (c),(d) Energy dependence of the ratio of two neighboring allowed harmonics $[I(3 q+2) / I(3 q+1)]$ at $\alpha=45^{\circ}$ for the measurements in (a) and (b). (e),(f) HHG spectra of $R$-MOX at the bicircular configuration (QWP angle $\alpha=45^{\circ}$ ) recorded under the experimental conditions reported in (c) and (d), respectively. The error bars in (c)-(f) represent 2 standard deviations of the measured signal.

chiral electronic dynamics induced by the strong driving fields. We return to this point in Sec. III B.

The angle-averaging procedure has the advantage of partially canceling the effects of polarization impurities. Residual imperfections lead to an asymmetric intensity distribution for the bielliptical fields of opposite handedness (Fig. S2 of SM [41]). However, the averaging procedure also reduces the maximum of $\mathrm{CD}(n \omega, \alpha)$ as a function of $\alpha$. The nonaveraged quantity is presented in Figs. S3 and S6 (SM) [41]. While the averaged $\mathrm{CD}\left[\overline{\mathrm{CD}}^{ \pm}(n \omega)\right]$ varies in the range of $3 \pm 1 \%$ (at $20.7 \mathrm{eV}$ ) to $7.7 \pm 1.5 \%(30.3 \mathrm{eV})$, the maximal $\mathrm{CD}$ values reach up to $13 \pm 6 \%$.

Closer inspection of Figs. 3(e) and 3(f) indicates that $\overline{\mathrm{CD}}^{ \pm}(n \omega)$ does not vary smoothly as a function of the photon energy, but exhibits pronounced variations from one harmonic order to the next. This trend is most clearly observed in the "forbidden" $3 q$ harmonics, whereby $\overline{\mathrm{CD}}^{ \pm}(3 q \omega)$ assumes consistently lower values (by a factor of 1.5$)$ compared to the "allowed" harmonics. We attribute this trend to the shift of the CD maxima of the $3 q$ harmonics to regions of lower ellipticity [cf. Figs. 2(a) and 2(d)] discussed earlier. This behavior becomes progressively less pronounced on approaching the cutoff, which is in line with the reduction of the contrast in the suppression of the $3 q$ orders in this region [cf. spectra in Figs. S1(a) and S1(b) in the SM [41]].
Figure 4 introduces the principle of the bielliptical approach, which relies on locating the ellipticity $\epsilon_{\omega}=$ $-\epsilon_{2 \omega}$ that maximizes the high-harmonic signal for a given enantiomer in the region of small ellipticity values $\left|\epsilon_{\omega}\right| \lesssim 0.2$. In this approach, the polarizations of the two driving fields are chosen to be parallel to each other at zero ellipticity (linear polarization) as opposed to the perpendicular configuration imposed by the "in-line" setup chosen to realize the bicircular' scheme. Figure 4(a) shows the yields of $R$-MOX (blue), $S$-MOX (red), and a racemic mixture of the two enantiomers. Whereas the yield of the racemate maximizes at zero ellipticity, the yields from the two enantiopure samples maximize for nonzero and opposite values of the ellipticity. The circles represent measured high-harmonic intensities, whereas the full lines represent a Gaussian fit to the data points.

Figures 4(b) and 4(c) show the maximizing ellipticities $\epsilon_{1}=-\epsilon_{2}$ as a function of the photon energy for two different combinations of $I_{\omega}$ and $I_{2 \omega}$ given in the caption. The data in Fig. 4(c) extend to higher photon energies compared to Fig. 4(b) because of the higher driving intensities. In both panels, the maximizing ellipticities of the enantiopure samples display the expected symmetry with respect to zero ellipticitiy. In Fig. 4(b), the maximizing ellipticities are larger, up to $\sim 4.4 \pm 0.2 \%(17.6 \mathrm{eV})$, compared to Fig. 4(b), where all ellipticities remain well below $2 \%$. 


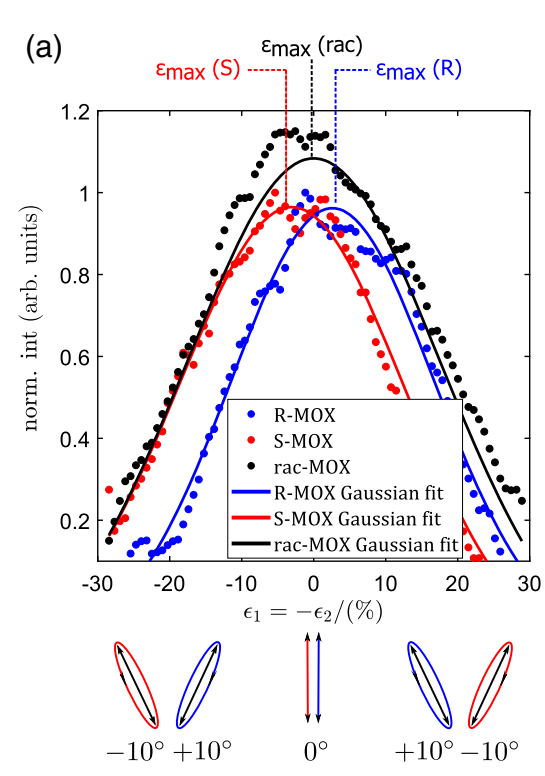

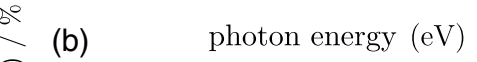
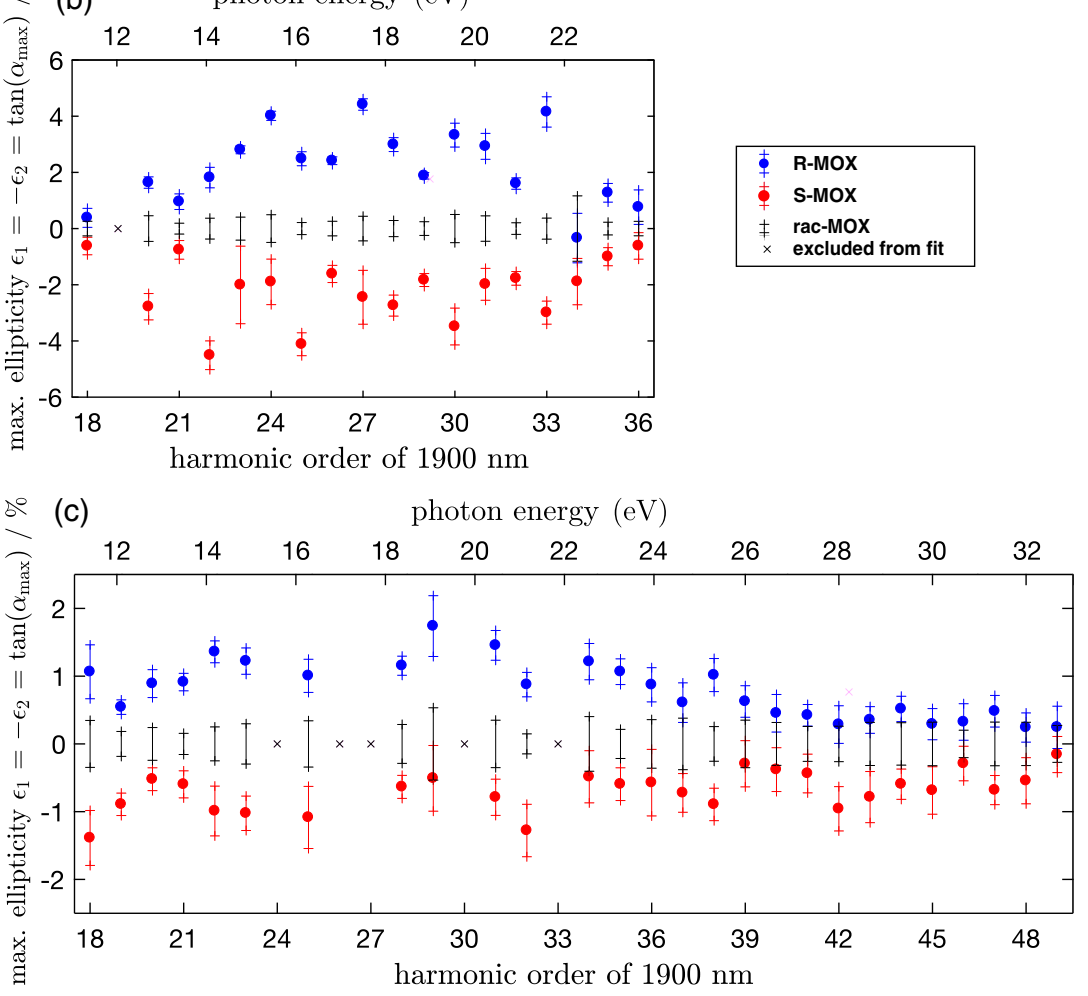

FIG. 4. Bielliptical high-harmonic spectroscopy. (a) HHG yield (data points) as a function of the driving ellipticity and Gaussian fits (full lines). The displacement of the maxima from $\alpha=0$ is a signature of the chiral response. The Lissajous figures indicate the polarization ellipses of the two drivers. The black points and curve have been shifted vertically for better visibility. The displayed data correspond to $H 28$ of $1900 \mathrm{~nm}$ recorded at $I_{\omega}=1.2 \times 10^{13} \mathrm{~W} / \mathrm{cm}^{2}$ and $I_{2 \omega}=1.8 \times 10^{13} \mathrm{~W} / \mathrm{cm}^{2}$. (b), (c) Maximizing ellipticity $\epsilon_{\max }$ as a function of the photon energy for two different intensities. (b) $I_{\omega}=1.2 \times 10^{13} \mathrm{~W} / \mathrm{cm}^{2}, I_{2 \omega}=1.8 \times 10^{13} \mathrm{~W} / \mathrm{cm}^{2}$ and (c) $I_{\omega}=1.5 \times 10^{13} \mathrm{~W} / \mathrm{cm}^{2}, I_{2 \omega}=2.1 \times 10^{13} \mathrm{~W} / \mathrm{cm}^{2}$. The error bars in (b) and (c) represent the $95 \%$ confidence interval obtained from the fitting procedure.

These results demonstrate a second experimental approach to detect molecular chirality using BHHS. As in the case of the bicircular scheme, the observed signatures are found to display a large intensity dependence, which again points to the central role of electronic dynamics in the chiral response.

\section{DISCUSSION}

\section{A. Mechanisms of chiral discrimination}

In the following, we discuss possible mechanisms underlying the chiral discrimination observed in our experiments. Our analysis closely follows the theoretical procedure outlined in Ref. [27]. Since HHG is only phase matched along the propagation direction of the driving beams, the present measurements lack an observable sensitive to the forward-backward asymmetry, which rules out a mechanism based solely on electric-dipole interactions (as in PECD measurements). This implies the participation of the magnetic laser field component [27], which can manifest itself in two ways: first, by means of the Lorentz force deflecting the electron trajectory out of the plane perpendicular to the field propagation axis, and second, through the interplay of electric- and magneticdipole transitions between the cationic states during the transit of the electron in the continuum [27].

Regarding the first mechanism, which has so far not been studied for the case of bicircular driving fields, the results of our analysis presented in the SM (Sec. III) [41] suggest that at the intensities employed in the experiment, its role is negligible as the predicted chiroptical response lies 2 orders of magnitude below the experimental outcome. These conclusions are similar to the findings for the case of single-color weakly elliptical fields [27] and suggest that the second mechanism dominates in inducing the chiral response. Our observation of a pronounced intensity dependence of the chiral response in Figs. 3(e), 3(f), and 4 lend further support to this interpretation. The next section summarizes the results of our extended theoretical analysis of this second mechanism. Compared to Refs. [26,37], our model uses a more accurate description of the photorecombination step based on electron-molecule quantum scattering calculations, as in our work on rare-gas atoms [35]. Our present results show that a good agreement 
between experiment and theory can also be achieved for much more complex systems, i.e., chiral molecules. This agreement is an important conclusion of our work that validates the theoretically predicted origin $[26,37,38]$ of the circular dichroism in bicircular driving fields.

\section{B. Theoretical model of chiral discrimination}

In this section, we outline our approach to modeling BHHG from chiral media. The strong-field-ionization step at the employed laser intensities can lead to the population of two states of the $\mathrm{C}_{3} \mathrm{H}_{6} \mathrm{O}^{+}$cation $(\tilde{X}$ and $\tilde{A}$ with vertical ionization potentials of 10.22 and $11.12 \mathrm{eV}$ ). During the electron excursion in the continuum, the strong laser field can transfer population between the two cationic states $\tilde{X}$ and $\tilde{A}$ through electric-dipole, magnetic-dipole, or electricquadrupole transitions. By symmetry, only the magneticdipole transitions can contribute to the $\mathrm{CD}$ in randomly oriented molecules (for details, see SM, Sec. IV [41]). In the final step, the electron can recombine with the ion in either of these eigenstates. The various ionizationrecombination paths [also referred to as "channels," cf. Fig. 5(a)] realized during the HHG process will be described by the nomenclature $I F,\{I, F\} \in\{X, A\}$, where $I$ is the initially populated state and $F$ is the cationic state to which recombination occurs. The appearance of "cross channels" $X A$ and $A X$ is solely due to the laser-induced population transfer between $\tilde{X}$ and $\tilde{A}$.

The HHG amplitude associated with the contribution of the channel $I F$ can be formulated by performing a decomposition of the frequency-domain HHG emission dipole $\boldsymbol{d}_{\text {tot }}$ into a product of the matrix elements pertaining to each individual step,

$$
\boldsymbol{d}_{I F}(n \omega) \propto \frac{1}{\sqrt{i}} a_{\mathrm{ion}}^{I} a_{\mathrm{prop}}^{\tilde{I}} \boldsymbol{a}_{\mathrm{rec}}^{F} b_{I F},
$$

where $b_{I F}$ are the coefficients describing the population transfer between the $\tilde{X}$ and $\tilde{A}$ states. The matrix elements $a_{\text {ion }}, a_{\text {prop }}, \boldsymbol{a}_{\text {rec }}$ are calculated on the basis of complex-valued quantum trajectories resulting from the saddle-point analysis of the HHG response. The electronic-structure calculations are performed at the multireference-configurationinteraction (MRCI) level following an equally weighted two-state-averaged complete-active-space self-consistentfield (CASSCF) calculation with 7 electrons in 6 active orbitals. The calculation of photorecombination matrix elements in the molecular frame are obtained from quantum scattering calculations based on the Schwinger variational principle [49,50]. Equation (4) is evaluated in the molecular frame and subsequently coherently averaged over an Eulerangle grid with $72 \times 72 \times 72$ points. Further details are given in the SM (Sec. IV) [41].

The results of our calculations for two intensities matching the experimental conditions are compared to the observed ratio curves in Fig. 5(b). The ratios are qualitatively well reproduced, especially at low orders and the local minima at $q=11$. The lack of quantitative agreement is not surprising considering the complexity of the system and the remaining approximations made in our model. In Fig. 5(c), the calculated CD is compared to the experimental values. The latter are obtained by integrating the ellipticity-dependent HHG intensity over the narrow range $\left(\alpha= \pm 40^{\circ}- \pm 50^{\circ}\right)$. The very encouraging result is the agreement in the magnitude of the effect, the calculation predicting $\mathrm{CD}$ values up to $\sim 7 \%$ without any adjustable parameter (except for the relative phase $\phi$ at ionization discussed below). The predicted magnitude of the CD thus lies close to the experimentally observed values in the energy region $n \sim 30-32$. Most remarkably, the intensity dependence of the $\mathrm{CD}$ is also correctly reproduced, decreasing by a factor of $\sim 2$ when the ratio of the driving fields $\left(\eta=E_{2 \omega} / E_{\omega}\right)$ is changed from $\eta \approx 0.6$ to $\eta \approx 0.5$.

We now turn to the dependence of the ratio and the CD on the shape of the electron hole created in the ionization step [30]. Figures 5(d) and 5(e) show the calculated results assuming different relative phases $\phi$ in the electronic superposition state created at ionization (defined as $t=0$ ):

$$
\Psi(t=0)=c_{\tilde{X}}(t=0) \psi_{\tilde{X}}+c_{\tilde{A}}(t=0) \psi_{\tilde{A}} e^{i \phi} .
$$

Both the intensity ratio and the $\mathrm{CD}$ are found to be sensitive to the initial phase of the superposition state, i.e., the shape of the initial hole. The best agreement is obtained for $\phi \approx-\pi / 8$, whereas values of $\phi$ that are much larger in magnitude can be excluded.

With the knowledge of the relative phase at the time of ionization, we can reconstruct the evolution of the electronhole density in the cation as a function of time. The result obtained by employing the calculated HOMO and HOMO-1 canonical Hartree-Fock orbitals, the initial phase $\phi=-\pi / 8$ estimated from the comparison with the experimental results and the time dependencies of $c_{\tilde{X}}(t)$ and $c_{\tilde{A}}(t)$ resulting from electric- and magnetic-dipole transitions, is shown in the top panel of Fig. 6. We note that the displayed hole dynamics is not per se responsible for the measured circular dichroism because the random orientation of the molecules cancels this possible contribution. Instead, it is the modification of the hole dynamics by the magneticdipole coupling that causes the chiral response. Since the influence of the magnetic field on the hole dynamics is very small, we visualize the effect underlying the $C D$ by representing differential hole densities. The bottom panel of Fig. 6 shows the differences between hole densities obtained in the presence and in the absence of magneticdipole coupling. Although the relative differences are on the order of $10^{-4}$, this small deviation underlies the large chiral response observed in our experiments. This is the consequence of the coherent amplification realized through the interference of the emission from the two channels $A X$ and 


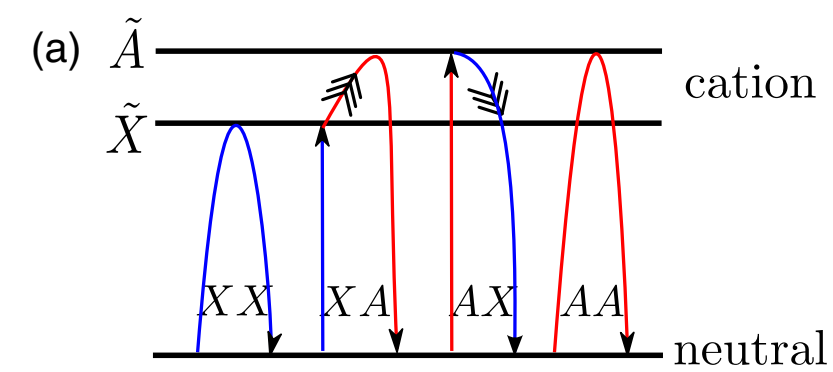

(c)
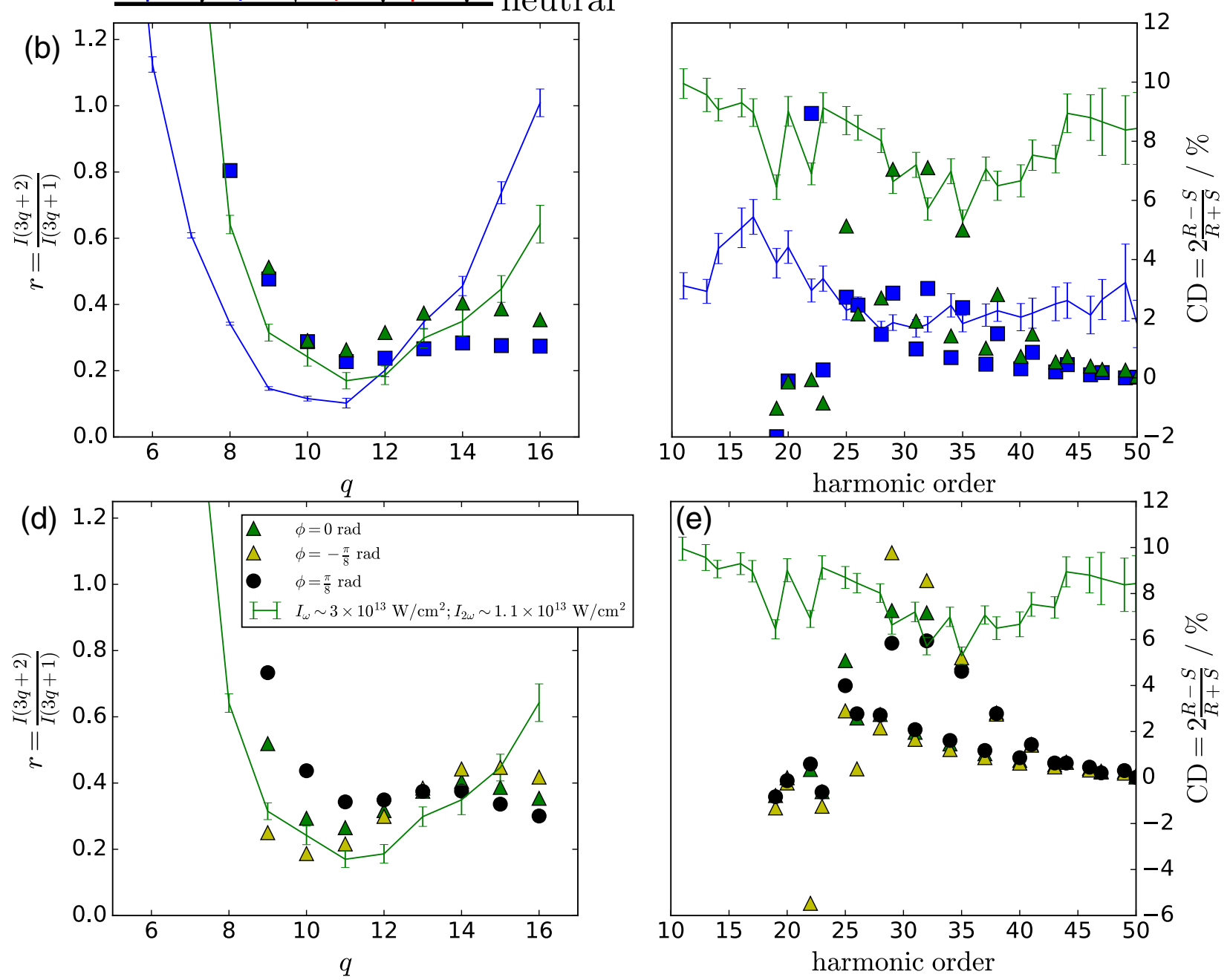

$\begin{array}{|ll|}\square & I_{\omega} \sim 3 \times 10^{13} \mathrm{~W} / \mathrm{cm}^{2} ; I_{2 \omega} \sim 0.8 \times 10^{13} \mathrm{~W} / \mathrm{cm}^{2} \text { (calc.) } \\ \Delta & I_{\omega} \sim 3 \times 10^{13} \mathrm{~W} / \mathrm{cm}^{2} ; I_{2 \omega} \sim 1.1 \times 10^{13} \mathrm{~W} / \mathrm{cm}^{2} \text { (calc.) } \\ \mathrm{F}-I_{\omega} \sim 3 \times 10^{13} \mathrm{~W} / \mathrm{cm}^{2} ; I_{2 \omega} \sim 0.75 \times 10^{13} \mathrm{~W} / \mathrm{cm}^{2} \\ \mathrm{I} & I_{\omega} \sim 3 \times 10^{13} \mathrm{~W} / \mathrm{cm}^{2} ; I_{2 \omega} \sim 1.1 \times 10^{13} \mathrm{~W} / \mathrm{cm}^{2}\end{array}$

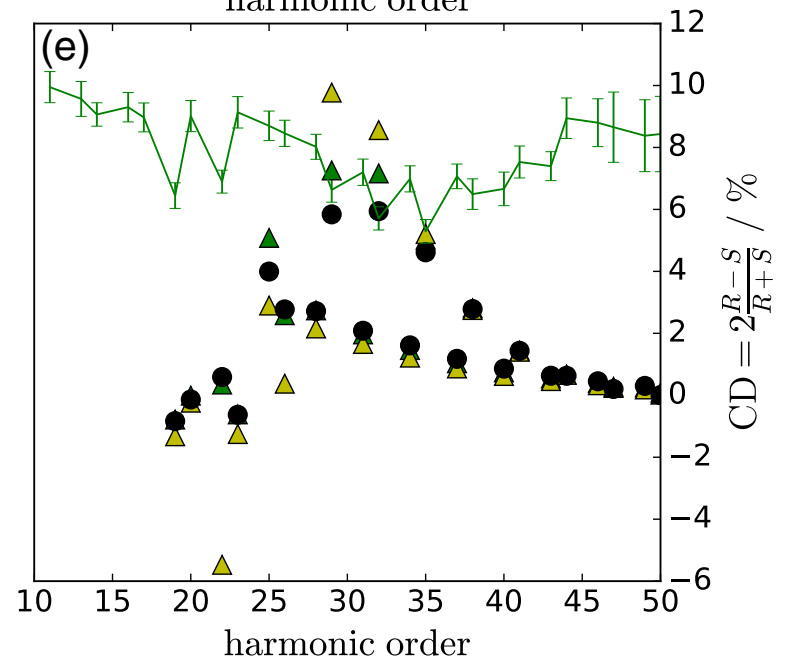

FIG. 5. Comparison of predicted and observed CD in BHHS. (a) Schematic illustration of the four ionization-recombination paths considered in the model. (b) Comparison (experiment versus theory) of the intensity ratios between two neighboring allowed harmonics at two different experimental conditions (cf. legends). (c) Energy dependence of the calculated CD: theory (markers) versus experiment (solid lines). (d) and (e) Calculated result for the intensity ratios (d) and the CD degree (e) using three different values of the initial phase $\phi$ between the two cationic states.

$X A$ carrying the chiral response, with the two remaining channels. A similar coherent amplification mechanism has been observed in HHG from a superposition of two electronic states of a neutral molecule (NO) [51].

We note that our present model does not capture the CD increase towards the cutoff region at $n \gtrsim 40$. This might be attributed to the divergence of the saddle-point solutions in the cutoff region where trajectory pairs start to coalesce. An additional source of error is the magnitude of the magnetic transition dipole moment, since this quantity determines the chiral response (see SM, Sec. 4.6 [41]). Nevertheless, our theoretical results are in better agreement with our experimental results compared to earlier predictions of the CD ( $\sim 20 \%$ at $|\epsilon| \approx 1$ ) [26], obtained within the eikonal-Volkov approximation of the recombination amplitude $\left(\boldsymbol{a}_{\text {rec }}\right)$, and also compared to the values of $<3 \%$ predicted with the use of photorecombination matrix elements from densityfunctional theory [37]. 


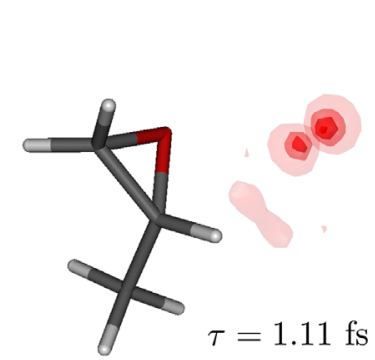

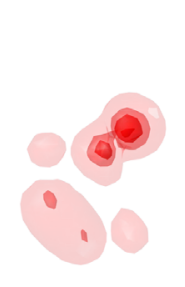

$1.46 \mathrm{fs}$
$\left|\Psi_{\text {hole }}(t)\right|^{2}$

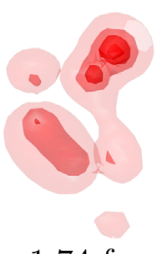

$1.74 \mathrm{fs}$

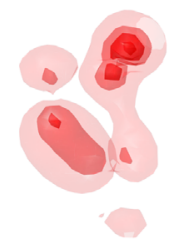

$2.03 \mathrm{fs}$

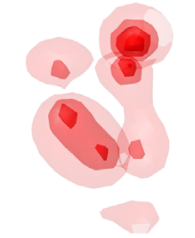

$2.33 \mathrm{fs}$
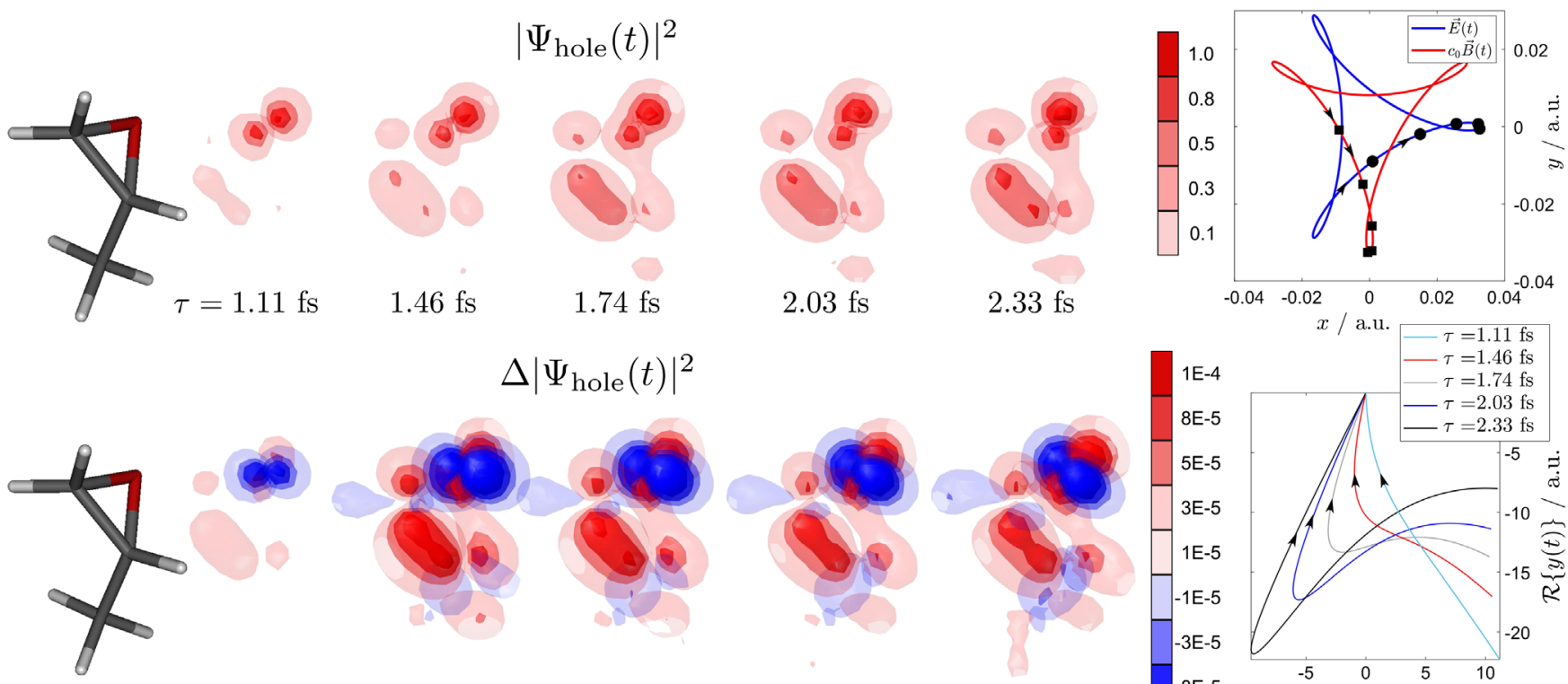

$\tau=1.11 \mathrm{fs}$

$1.46 \mathrm{fs}$

$1.74 \mathrm{fs}$

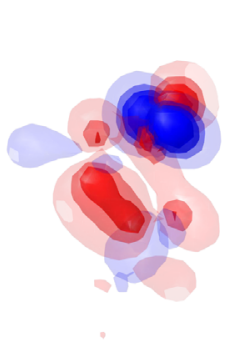

$2.03 \mathrm{fs}$

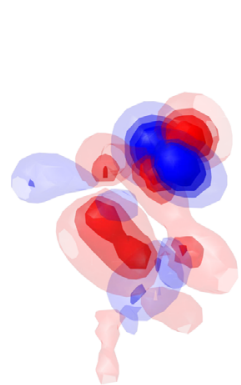

$2.33 \mathrm{fs}$
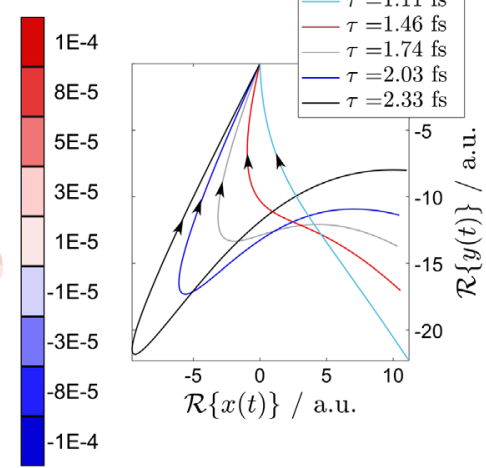

FIG. 6. Electron-hole dynamics underlying the chiral response. These simulations were done for laser parameters matching the experimental results in Fig. $1\left(1800\right.$ and $\left.900 \mathrm{~nm}, I_{\omega}=3.0 \times 10^{13} \mathrm{~W} / \mathrm{cm}^{2}, I_{2 \omega}=1.1 \times 10^{13} \mathrm{~W} / \mathrm{cm}^{2}\right)$ and $\phi=-\pi / 8$, as estimated by comparison with the experimental results. Top row: Absolute magnitude of the hole density at the time of recombination for 5 harmonic orders $(H 17,24,32,40$, and 48). The corresponding transit times of the continuum electron $\tau$ are indicated. Bottom row: Difference between the hole densities calculated with and without the contribution of the magnetic-dipole interaction. The contour levels are coded in the colors of the isocontour surfaces (color bar on the right). Top right: Spatial profiles of the electric (blue) and the magnetic (red) fields. The ionization times of the five trajectories are indicated as black circles (electric field) or black squares (magnetic field), respectively. Bottom right: Real part of the spatial quantum trajectories.

\section{CONCLUSION}

We present two variants of BHHS for detecting a strong chiroptical response in an ensemble of randomly oriented gas-phase molecules. Combining two counterrotating pulses of different frequencies and tunable polarization states provides many rich opportunities for controlling the degree of $\mathrm{CD}$ by tuning the driving wavelength, the relative intensity, and the polarization of the two field components. The bicircular technique leads to relatively high levels of $\mathrm{CD}$ of up to $13 \%$ whereas the bielliptical technique leads to maximizing ellipticities of up to $\sim 4 \%$. Both techniques thus achieve a higher degree of chiral discrimination than singlecolor elliptically polarized HHG [27]. Detailed measurements of the high-harmonic yields and circular dichroism as a function of the driving-field ellipticities have revealed a characteristic dependence on the "allowed" $(3 q)$ or "forbidden" nature $(3 q \pm 1)$ of the harmonic orders. These effects are shown to originate from the interference between the emissions of three quantum trajectories, which become inequivalent when the driving fields deviate from circularity. All chiral observables were found to sensitively depend on the intensities of the driving fields. These observations are a direct experimental indication that the chiral response originates from laser-driven dynamics in the molecular cation. This assertion was fully validated by a complete theoretical model of BHHS in bicircular driving fields, including complex-valued quantum trajectories, accurate electronic-structure calculations, and quantitative photorecombination matrix elements obtained from molecular quantum scattering calculations. On the basis of this model, a good agreement was achieved between measured and calculated intensity ratios of neighboring harmonics as well as on the magnitude of the CD effect. This level of agreement, paired with the correct reproduction of the intensity dependence of the chiral dichroism, supports theoretical predictions for BHHG, which identified chiral electron dynamics in $\mathrm{MOX}^{+}$as being the dominant effect behind the chiral discrimination. Our work has established a pronounced sensitivity of the observables to the relative phase of the electronic states of $\mathrm{MOX}^{+}$ prepared in the ionization step. This has allowed us to relate the measured chiral effects to the chiral hole dynamics of $\mathrm{MOX}^{+}$and to theoretically investigate the influence of the magnetic-dipole coupling on the hole dynamics. Although the results in this work focus on MOX, the technique is expected to be completely general because multiple electronic states of the cation will contribute to HHG in all chiral molecules and magnetic-dipole transitions between them are always allowed by symmetry. Owing to its ultrafast timescale, this method offers the prospect for probing chiral dynamics in a pump-probe scheme. The 
demonstrated sensitivity of the BHHS spectra to the initial phase of the electronic superposition state prepared in the cation opens the path to the study of magnetic-fieldcontrolled attosecond charge migration in chiral molecules, offering an additional, chirality-specific control aspect to previous work [30].

\section{ACKNOWLEDGMENTS}

We thank H. Herburger and S. Brennecke for their contributions to the experimental part of the project. We acknowledge financial support from an ERC Starting Grant (Contract No. 307270-ATTOSCOPE) and the National Centre of Competence in Research-Molecular Ultrafast Science and Technology (NCCR-MUST), a funding instrument of the Swiss National Science Foundation. We thank the Euler high-performance computing cluster of the ETH Zurich for allocation of computational resources.

\section{APPENDIX: EXPERIMENTAL METHODS}

The experimental setup comprises an amplified femtosecond Ti:sapphire laser system and a vacuum chamber for the generation and spectral analysis of high-harmonic radiation. A portion $(5.8-6 \mathrm{~mJ})$ of the output of the laser system $(\approx 11.5 \mathrm{~mJ}, 800 \mathrm{~nm}, \approx 30 \mathrm{fs}, 1 \mathrm{kHz})$ is used to pump an optical parametric amplifier (TOPAS-HE, Light Conversion). We use the idler beam (pulse energy of 0.9$1 \mathrm{~mJ}$ and duration of $\approx 45 \mathrm{fs}$ ) with central wavelength tuned to either 1800 or $1900 \mathrm{~nm}$. The pulse is frequency doubled in a BBO type-I crystal of thickness $250 \mu \mathrm{m}$ or $1 \mathrm{~mm}$, enabling conversion efficiencies from $\approx 18 \%$ to $\gtrsim 50 \%$. Two alternative setups are used to convert the orthogonally polarized fundamental and second harmonic into a bicircular field.

The "bicircular" scheme is realized in an "in-line" setup described in detail in Ref. [52]. After the BBO, the two linearly polarized orthogonal driving fields are temporally synchronized by a pair of calcite plates $(2 \times 600 \mu \mathrm{m})$, that precompensate the different group delay dispersions accumulated in the BBO and the additional dispersive optics in the remaining part of the beam paths, and pass a broadband achromatic quarter-wave plate. The major axis orientation of the latter is gradually varied from $-60^{\circ}$ to $+60^{\circ}$, thereby converting the polarization of the two beams from circular $\left(\alpha=-45^{\circ}\right)$, where the $\omega$ component is left circularlypolarized (LCP) and the $2 \omega$ is right circularly-polarized (RCP), via elliptical to linear $(\alpha=0$, with the two polarization axes being orthogonal to each other), and finally to circular at $\alpha=+45^{\circ}$ (of the opposite helicity, i.e., with RCP $\omega$ and LCP $2 \omega$ ).

The "bielliptical" scheme is realized in a Mach-Zehnder interferometric setup [32-35], whereby the two frequency components are separated and later recombined with the help of a dichroic mirror pair (HT 1800-2000 nm, HR 900$1000 \mathrm{~nm})$. The polarization of each beam is varied independently with the aid of a $\lambda / 2+\lambda / 4$ wave plate pair located in each arm. The relative $\omega-2 \omega$ delay between the pulses is controlled with the help of an ultraprecise $( \pm 0.2 \mu \mathrm{m}$ accuracy) translation stage (XMS50, Newport) in the $2 \omega$ arm.

In both cases, the bicircular beam is focused into a vacuum chamber with the help of a silver mirror with $f=35 \mathrm{~cm}$. The chamber window material is $\mathrm{CaF}_{2}$ or ultraviolet-grade fused silica and has a thickness of $1 \mathrm{~mm}$. The focused two-color laser beam intersects a thin molecular beam of enantiopure methyl oxirane seeded in helium. Under the chosen laser intensities, no emission from helium is detected. The harmonic radiation is dispersed on a grating with 1200 lines $\mathrm{mm}^{-1}$ or 300 lines $\mathrm{mm}^{-1}$ and detected on a microchannel plate detector backed up with phosphor screen and imaged on a CCD camera.

Approximately $3-5 \mathrm{~mL}$ of neat liquid methyl oxirane (enantiopure $R$ - or $S$-MOX, GC $>98 \%$, Tokyo Chemical Industry, or racemic $R / S$-MOX, $\geq 99.5 \%$, Aldrich-Fine Chemicals) are loaded into stainless-steel vessels connected to a gas delivery system that enables rapid switching between the samples. A continuous flow of helium carrier gas at a backing pressure of $0.8-1$ bar is passed through the liquid samples kept at room temperature, thereby delivering the vapor to the nozzle of a pulsed valve operating at $100 \mathrm{~Hz}$. The gas is subsequently injected into the vacuum via a $500 \mu \mathrm{m}$ orifice. The vapor pressure of MOX is sufficiently high at standard conditions to yield a pressure of $\approx 10^{-4}$ mbar in the source chamber, as measured with a Pfeiffer Vacuum pressure gauge. In order to prevent potential condensation, the pipes of the delivery manifold as well as the nozzle itself are kept at a slightly elevated temperature of $35^{\circ} \mathrm{C}-40^{\circ} \mathrm{C}$. (For comparison, the boiling temperature of $\mathrm{MOX}$ is $34^{\circ} \mathrm{C}$ at atmospheric pressure.) The laser intersects the thin gas jet $\approx 1 \mathrm{~mm}$ below the nozzle. The estimated length of the interaction region is thus much smaller than the Rayleigh length. This choice minimizes the influence of macroscopic effects on the high-harmonic emission. Moreover, owing to their longer transit times, the "long" trajectories in bicircular fields are sufficiently more suppressed than the long trajectories in linearly polarized single-color fields that they play no role in our measurements.

[1] L. D. Barron, Molecular Light Scattering and Optical Activity, 2nd ed. (Cambridge University Press, New York, 2004).

[2] E. Castiglioni, S. Abbate, and G. Longhi, Experimental Methods for Measuring Optical Rotatory Dispersion: Survey and Outlook, Chirality 23, 711 (2011).

[3] L. D. Barron and A. D. Buckingham, Vibrational Optical Activity, Chem. Phys. Lett. 492, 199 (2010).

[4] P. W. Atkins and L.D. Barron, Rayleigh Scattering of Polarized Photons by Molecules, Mol. Phys. 16, 453 (1969). 
[5] L. D. Barron and A. D. Buckingham, Rayleigh and Raman Scattering from Optically Active Molecules, Mol. Phys. 20, 1111 (1971).

[6] L. D. Barron, M. P. Bogaard, and A. D. Buckingham, Raman Scattering of Circularly Polarized Light by Optically Active Molecules, J. Am. Chem. Soc. 95, 603 (1973).

[7] Y. Inoue and V. Ramamurthy, Chiral Photochemistry (Marcel Dekker, New York, 2004), p. 704.

[8] L. Rosenfeld, Quantenmechanische Theorie der Natürlichen Optischen Aktivität von Flüssigkeiten und Gasen, Z. Phys. 52, 161 (1929).

[9] D. Patterson, M. Schnell, and J. M. Doyle, EnantiomerSpecific Detection of Chiral Molecules via Microwave Spectroscopy, Nature (London) 497, 475 (2013).

[10] M. Pitzer, M. Kunitski, A. S. Johnson, T. Jahnke, H. Sann, F. Sturm, L. P. H. Schmidt, H. Schmidt-Böcking, R. Dörner, J. Stohner, J. Kiedrowski, M. Reggelin, S. Marquardt, A. Schiesser, R. Berger, and M. S. Schöffler, Direct Determination of Absolute Molecular Stereochemistry in Gas Phase by Coulomb Explosion Imaging, Science 341, 1096 (2013).

[11] P. Herwig, K. Zawatzky, M. Grieser, O. Heber, B. JordonThaden, C. Krantz, O. Novotný, R. Repnow, V. Schurig, D. Schwalm, Z. Vager, A. Wolf, O. Trapp, and H. Kreckel, Imaging the Absolute Configuration of a Chiral Epoxide in the Gas Phase, Science 342, 1084 (2013).

[12] P. Herwig, K. Zawatzky, D. Schwalm, M. Grieser, O. Heber, B. Jordon-Thaden, C. Krantz, O. Novotný, R. Repnow, V. Schurig, Z. Vager, A. Wolf, O. Trapp, and H. Kreckel, Absolute Configuration Assignment of a Chiral Molecule in the Gas Phase Using Foil-Induced Coulomb Explosion Imaging, Phys. Rev. A 90, 052503 (2014).

[13] M. Pitzer et al., Absolute Configuration from Different Multifragmentation Pathways in Light-Induced Coulomb Explosion Imaging, ChemPhysChem 17, 2465 (2016).

[14] R. Li, R. Sullivan, W. Al-Basheer, R. M. Pagni, and R. N. Compton, Linear and Nonlinear Circular Dichroism

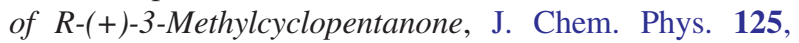
144304 (2006).

[15] A. Bornschlegl, C. Logé, and U. Boesl, Investigation of $C D$ Effects in the Multi Photon Ionisation of $R-(+)-3-$ Methylcyclopentanone, Chem. Phys. Lett. 447, 187 (2007).

[16] N. Böwering, T. Lischke, B. Schmidtke, N. Müller, T. Khalil, and U. Heinzmann, Asymmetry in Photoelectron Emission from Chiral Molecules Induced by Circularly Polarized Light, Phys. Rev. Lett. 86, 1187 (2001).

[17] B. Ritchie, Theory of the Angular Distribution of Photoelectrons Ejected from Optically Active Molecules and Molecular Negative Ions, Phys. Rev. A 13, 1411 (1976).

[18] I. Powis, Photoelectron Circular Dichroism in Gas Phase Chiral Molecules, Adv. Chem. Phys. 138, 267 (2008).

[19] G. A. Garcia, L. Nahon, M. Lebech, J. C. Houver, D. Dowek, and I. Powis, Circular Dichroism in the Photoelectron Angular Distribution from Randomly Oriented Enantiomers of Camphor, J. Chem. Phys. 119, 8781 (2003).

[20] C. Lux, M. Wollenhaupt, T. Bolze, Q. Liang, J. Köhler, C. Sarpe, and T. Baumert, Circular Dichroism in the Photoelectron Angular Distributions of Camphor and Fenchone from Multiphoton Ionization with Femtosecond Laser Pulses, Angew. Chem., Int. Ed. Engl. 51, 5001 (2012).
[21] C. S. Lehmann, N. B. Ram, I. Powis, and M. H. M. Janssen, Imaging Photoelectron Circular Dichroism of Chiral Molecules by Femtosecond Multiphoton Coincidence Detection, J. Chem. Phys. 139, 234307 (2013).

[22] C. Lux, M. Wollenhaupt, C. Sarpe, and T. Baumert, Photoelectron Circular Dichroism of Bicyclic Ketones from Multiphoton Ionization with Femtosecond Laser Pulses, ChemPhysChem 16, 115 (2015).

[23] S. Beaulieu, A. Comby, B. Fabre, D. Descamps, A. Ferré, G. Garcia, R. Géneaux, F. Légaré, L. Nahon, S. Petit, T. Ruchon, B. Pons, V. Blanchet, and Y. Mairesse, Probing Ultrafast Dynamics of Chiral Molecules Using TimeResolved Photoelectron Circular Dichroism, Faraday Discuss. 194, 325 (2016).

[24] A. Comby, S. Beaulieu, M. Boggio-Pasqua, D. Descamps, F. Légaré, L. Nahon, S. Petit, B. Pons, B. Fabre, Y. Mairesse, and V. Blanchet, Relaxation Dynamics in Photoexcited Chiral Molecules Studied by Time-Resolved Photoelectron Circular Dichroism: Toward Chiral Femtochemistry, J. Phys. Chem. Lett. 7, 4514 (2016).

[25] S. Beaulieu, A. Comby, A. Clergerie, J. Caillat, D. Descamps, N. Dudovich, B. Fabre, R. Géneaux, F. Légaré, S. Petit, B. Pons, G. Porat, T. Ruchon, R. Taïeb, V. Blanchet, and Y. Mairesse, Attosecond-Resolved Photoionization of Chiral Molecules, Science 358, 1288 (2017).

[26] O. Smirnova, Y. Mairesse, and S. Patchkovskii, Opportunities for Chiral Discrimination Using High Harmonic Generation in Tailored Laser Fields, J. Phys. B 48, 234005 (2015).

[27] R. Cireasa, A. E. Boguslavskiy, B. Pons, M. C. H. H Wong, D. Descamps, S. Petit, H. Ruf, N. Thiré, A. Ferré, J. Suarez, J. Higuet, B. E. Schmidt, A. F. Alharbi, F. Légaré, V. Blanchet, B. Fabre, S. Patchkovskii, O. Smirnova, Y. Mairesse, and V. R. Bhardwaj, Probing Molecular Chirality on a SubFemtosecond Timescale, Nat. Phys. 11, 654 (2015).

[28] S. Baker, J. S. Robinson, C. A. Haworth, H. Teng, R. A. Smith, C. C. Chirila, M. Lein, J. W. G. Tisch, and J. P. Marangos, Probing Proton Dynamics in Molecules on an Attosecond Time Scale, Science 312, 424 (2006).

[29] O. Smirnova, S. Patchkovskii, Y. Mairesse, N. Dudovich, D. Villeneuve, P. Corkum, and M. Y. Ivanov, Attosecond Circular Dichroism Spectroscopy of Polyatomic Molecules, Phys. Rev. Lett. 102, 063601 (2009).

[30] P. M. Kraus, B. Mignolet, D. Baykusheva, A. Rupenyan, L. Horný, E. F. Penka, G. Grassi, O. I. Tolstikhin, J. Schneider, F. Jensen, L. B. Madsen, A. D. Bandrauk, F. Remacle, and H. J. Wörner, Measurement and Laser Control of Attosecond Charge Migration in Ionized Iodoacetylene, Science 350, 790 (2015).

[31] H. Eichmann, A. Egbert, S. Nolte, C. Momma, B. Wellegehausen, W. Becker, S. Long, and J. K. McIver, Polarization-Dependent High-Order Two-Color Mixing, Phys. Rev. A 51, R3414 (1995).

[32] A. Fleischer, O. Kfir, T. Diskin, P. Sidorenko, and O. Cohen, Spin Angular Momentum and Tunable Polarization in High-Harmonic Generation, Nat. Photonics 8, 543 (2014).

[33] O. Kfir, P. Grychtol, E. Turgut, R. Knut, D. Zusin, D. Popmintchev, T. Popmintchev, H. Nembach, J. M. Shaw, A. Fleischer, H. Kapteyn, M. Murnane, and O. Cohen, Generation of Bright Circularly-Polarized Extreme 
Ultraviolet High Harmonics for Magnetic Circular Dichroism Spectroscopy, Nat. Photonics 9, 99 (2015).

[34] D. Baykusheva, M. S. Ahsan, N. Lin, and H. J. Wörner, Bicircular High-Harmonic Spectroscopy Reveals Dynamical Symmetries of Atoms and Molecules, Phys. Rev. Lett. 116, 123001 (2016).

[35] D. Baykusheva, S. Brennecke, M. Lein, and H. J. Wörner, Signatures of Electronic Structure in Bicircular HighHarmonic Spectroscopy, Phys. Rev. Lett. 119, 203201 (2017).

[36] Á. Jiménez-Galán, N. Zhavoronkov, M. Schloz, F. Morales, and M. Ivanov, Time-Resolved High Harmonic Spectroscopy of Dynamical Symmetry Breaking in Bi-Circular Laser Fields, Opt. Express 25, 22880 (2017).

[37] D. Ayuso, P. Decleva, S. Patchkovskii, and O. Smirnova, Chiral Dichroism in Bi-Elliptical High-Order Harmonic Generation, J. Phys. B 51, 06LT01 (2018).

[38] D. Ayuso, P. Decleva, S. Patchkovskii, and O. Smirnova, Strong-Field Control and Enhancement of Chiral Response in Bi-Elliptical High-Order Harmonic Generation: An Analytical Model, J. Phys. B 51, 124002 (2018).

[39] A. Fleischer, E. Bordo, O. Kfir, G. Ilan, P. Sidorenko, and O. Cohen, High Harmonic Generation Polarization States: Circular, Linear and Foucault-Fan, in Proceedings of the 6th International Conference on Attosecond Physics, 2017.

[40] Y. Harada, E. Haraguchi, K. Kaneshima, and T. Sekikawa, Circular Dichroism in High-Order Harmonic Generation from Chiral Molecules, Phys. Rev. A 98, 021401 (2018).

[41] See Supplemental Material at http://link.aps.org/ supplemental/10.1103/PhysRevX.8.031060 for details on the data analysis procedure, additional experimental data and further details on the theoretical calculations.

[42] D. B. Milošević, W. Becker, and R. Kopold, Generation of Circularly Polarized High-Order Harmonics by Two-Color Coplanar Field Mixing, Phys. Rev. A 61, 063403 (2000).

[43] H. J. Wörner, H. Niikura, J. B. Bertrand, P. B. Corkum, and D. M. Villeneuve, Observation of Electronic Structure Minima in High-Harmonic Generation, Phys. Rev. Lett. 102, 103901 (2009).
[44] O. Smirnova, Y. Mairesse, S. Patchkovskii, N. Dudovich, D. M. Villeneuve, P. B. Corkum, and M. Y. Ivanov, High Harmonic Interferometry of Multi-Electron Dynamics in Molecules, Nature (London) 460, 972 (2009).

[45] H. J. Wörner, J. B. Bertrand, P. Hockett, P. B. Corkum, and D. M. Villeneuve, Controlling the Interference of Multiple Molecular Orbitals in High-Harmonic Generation, Phys. Rev. Lett. 104, 233904 (2010).

[46] A. Rupenyan, P. M. Kraus, J. Schneider, and H. J. Wörner, High-Harmonic Spectroscopy of Isoelectronic Molecules: Wavelength Scaling of Electronic-Structure and Multielectron Effects, Phys. Rev. A 87, 033409 (2013).

[47] A. Rupenyan, P. M. Kraus, J. Schneider, and H. J. Wörner, Quantum Interference and Multielectron Effects in HighHarmonic Spectra of Polar Molecules, Phys. Rev. A 87, 031401 (2013).

[48] K. M. Dorney, J. L. Ellis, C. Hernández-García, D. D. Hickstein, C. A. Mancuso, N. Brooks, T. Fan, G. Fan, D. Zusin, C. Gentry, P. Grychtol, H. C. Kapteyn, and M. M. Murnane, Helicity-Selective Enhancement and Polarization Control of Attosecond High Harmonic Waveforms Driven by Bichromatic Circularly Polarized Laser Fields, Phys. Rev. Lett. 119, 063201 (2017).

[49] F. A. Gianturco, R. R. Lucchese, and N. Sanna, Calculation of Low-Energy Elastic Cross Sections for Electron- $\mathrm{CF}_{4}$ Scattering, J. Chem. Phys. 100, 6464 (1994).

[50] A. Natalense and R. R. P. P. Lucchese, Cross Section and Asymmetry Parameter Calculation for Sulfur 1s Photoionization of $\mathrm{SF}_{6}$, J. Chem. Phys. 111, 5344 (1999).

[51] P. M. Kraus, S. B. Zhang, A. Gijsbertsen, R. R. Lucchese, N. Rohringer, and H. J. Wörner, High-Harmonic Probing of Electronic Coherence in Dynamically Aligned Molecules, Phys. Rev. Lett. 111, 243005 (2013).

[52] O. Kfir, E. Bordo, G. I. Haham, O. Lahav, A. Fleischer, and O. Cohen, In-Line Production of a Bi-Circular Field for Generation of Helically Polarized High-Order Harmonics, Appl. Phys. Lett. 108, 211106 (2016). 